\title{
Ultrasound and microbubble-mediated targeted delivery of therapeutic microRNA-loaded nanocarriers to deep liver and kidney tissues in pigs
}

Tommaso Di lanni, Rajendran J.C. Bose, Uday K. Sukumar, Sunitha Bachawal,

Huaijun Wang, Arsenii Telichko, Carl Herickhoff, Elise Robinson,

Sam Baker, José G. Vilches-Moure, Stephen A. Felt, Sanjiv S. Gambhir,

Ramasamy Paulmurugan, Jeremy D. Dahl

The manuscript was withdrawn per the authors' request. 